\title{
Kinetic Microparticle Immunoassay
}

National Cancer Institute

\section{Source}

National Cancer Institute. Kinetic Microparticle Immunoassay. NCI Thesaurus. Code C111240.

A type of immunoassay in which the antigen of interest found in a biological specimen competes with a lattice of antigen-antibody complexes. Competing antigen inhibits lattice formation and decreases absorbance in proportion to the amount of antigen present in a biological specimen. 